\title{
Electronic states and persistent currents in nanowire quantum ring
}

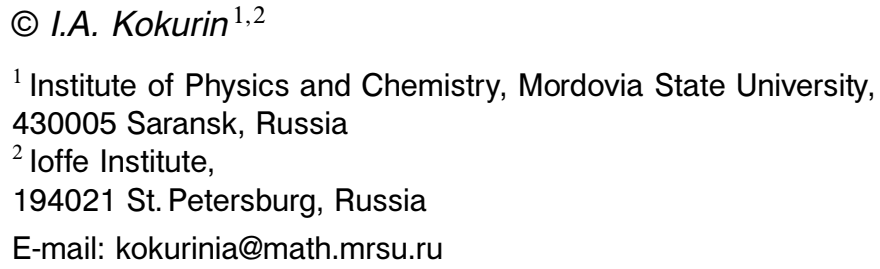

A new model of a quantum ring defined inside a nanowire is proposed. The one-particle Hamiltonian for electron in [111]-oriented nanowire quantum ring is constructed taking into account both Rashba and Dresselhaus spin-orbit coupling. The energy levels as a function of magnetic field are found using the exact numerical diagonalization. The persistent currents (both charge and spin) are calculated. The specificity of spin-orbit coupling and arising anticrossings in energy spectrum lead to unusual features in persistent current behavior. The variation of magnetic field or carrier concentration by means of gates can lead to pure spin persistent current with the charge current being zero. 